LAWRENCE LIVERMORE N A T IO N A L LABORATORY

Direct observation of the saturation of stimulated Brillouin scattering by ion-trapping induced frequency shifts

D. H. Froula, L. Divol, A. A. Offenberger, N. Meezan, T. Ao, G. Gregori, C. Niemann, D. Price, C. A. Smith, S. H. Glenzer

March 10, 2004

Physical Review Letters 
This document was prepared as an account of work sponsored by an agency of the United States Government. Neither the United States Government nor the University of California nor any of their employees, makes any warranty, express or implied, or assumes any legal liability or responsibility for the accuracy, completeness, or usefulness of any information, apparatus, product, or process disclosed, or represents that its use would not infringe privately owned rights. Reference herein to any specific commercial product, process, or service by trade name, trademark, manufacturer, or otherwise, does not necessarily constitute or imply its endorsement, recommendation, or favoring by the United States Government or the University of California. The views and opinions of authors expressed herein do not necessarily state or reflect those of the United States Government or the University of California, and shall not be used for advertising or product endorsement purposes. 


\title{
Direct observation of the saturation of stimulated Brillouin scattering by ion-trapping induced frequency shifts
}

\author{
D. H. Froula, L. Divol, A. A. Offenberger, ${ }^{*}$ N. Meezan, T. Ao, ${ }^{\dagger}$ G. \\ Gregori, C. Niemann, D. Price, C. A. Smith,$^{\ddagger}$ and S. H. Glenzer \\ L-399, Lawrence Livermore National Laboratory \\ University of California P. O. Box 808, CA 94551, U.S.A.
}

\begin{abstract}
We report the first measurement of the saturation of stimulated Brillouin scattering (SBS) by an ion-trapping induced frequency shift, which was achieved by directly measuring the amplitude and absolute frequency of SBS-driven ion-acoustic waves (IAW). A frequency shift of up to $30 \%$ and a simultaneous saturation of driven IAW and SBS reflectivity was observed. The scaling of the frequency shift with the IAW amplitude compares well with theoretical calculations. We have further measured fast 30 ps oscillations of the SBS-driven IAW amplitude induced by the frequency shift.

PACS numbers: 52.25.Qt, 52.35.Fp, 52.40.Nk, 52.50.Jm
\end{abstract}

Stimulated scattering is known to be capable of reflecting a large fraction of incident energy from inertial confinement fusion (ICF) targets [1-3]. It is therefore important to the success of ICF to understand the physical processes and to develop models $[4,5]$ that will predict plasma wave growth and stimulated scattering laser energy losses. Stimulated Brillouin scattering (SBS) is the result of the resonant ponderomotive coupling of an incident light wave, a reflected light wave, and an ion-acoustic wave. After an initial exponential growth, SBS driven ion-acoustic waves have been shown to saturate $[6,7]$. Among others, particle trapping and resulting frequency shifts have been suggested as a saturation mechanism in low-Z plasmas [8-12].

In this letter, we present the first measurement of a shift in the frequency of the ion-acoustic waves that are excited by SBS. The frequency shift directly explains the measured saturation of both the amplitude of the driven ion-acoustic wave and the SBS reflectivity. Our modeling indicates that large ion-acoustic waves trap ions, shifting the frequency of the ion-acoustic wave, resulting in a detuning of the SBS instability. Analytical calculations relate the measured frequency shift (up to $\left.\Delta \omega / \omega_{a}=-35 \%\right)$ to ion trapping. Furthermore, fast 30 ps amplitude modulations in the driven ion-acoustic wave have been measured for the first time. The frequency of the ion-acoustic wave modulation is set by the frequency shift that detunes the SBS ion-acoustic wave, as observed in a classical oscillator driven off its natural frequency. These measurements have been made possible by the use of two Thomson-scattering (TS) diagnostics which directly measure the absolute frequency and amplitude of the ion-acoustic wave responsible for SBS. Using one TS diagnostic to independently measure the ion-acoustic sound speed, the actual frequency shift responsible for saturating the SBS instability is measured with the second TS diagnostic.

The experiment used a three-beam configuration at the upgraded Janus Laser Facility at Lawrence Liver-

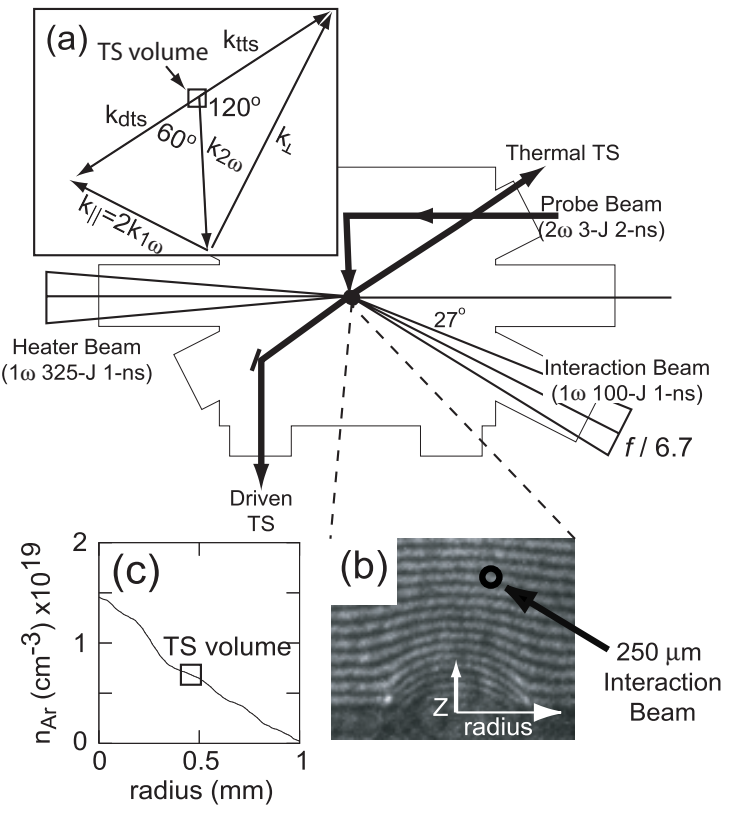

FIG. 1: The experimental setup is shown. All beams propagate in the plane of the figure. The gas target is emitted perpendicular to this plane. (a) A diagram detailing the ionacoustic wave vectors probed is shown. (b) A cylindrical density profile is measured from Abel inverting a two dimensional neutral density Ar interferometric image. The position of the interaction beam is represented by the superimposed circle $(\mathrm{r}=400 \mu \mathrm{m}, \mathrm{z}=1000 \mu \mathrm{m})$. (c) The radial density profile along the interaction beam path is shown. The TS volume is represented by the square.

more National Laboratory. The gas jet plasma has been well characterized for a variety of gases using a standard Mach-Zender interferometer $[13,14]$. An example of the Ar neutral density profile is shown in Fig. 1b. A 600-psi backing pressure was used with a 1-mm cylindrical nozzle to create a peak neutral density $1-\mathrm{mm}$ above the gas jet of $\mathrm{n}=1.5 \times 10^{19} \mathrm{~cm}^{-3}$ [Fig. 1c] corresponding to a peak electron density of $\mathrm{n}_{e}=3 \times 10^{19} \mathrm{~cm}^{-3}$ in our $\mathrm{He}$ 
plasmas.

The He gas jet plasmas were produced by a heater beam with $325 \mathrm{~J}$ of $1 \omega\left(\lambda_{1 \omega}=1054 \mathrm{~nm}\right)$ laser light in a 1.2-ns-long square pulse. The heater beam was focused 1$\mathrm{mm}$ above the gas jet using an $\mathrm{f} / 6.7$ lens and a phase zone plate (PZP) producing a $1.2-\mathrm{mm}$ diameter focal spot at target chamber center [Fig. 1]. A 1.2-ns $1 \omega$ interaction beam was used to drive the SBS process which excites ion-acoustic waves parallel to the interaction beam with a wave vector $\mathrm{k}_{\|}=2 \mathrm{k}_{1 \omega}$ [Fig. 1a]. The energy in the interaction beam was varied $10 \mathrm{~J}<\mathrm{E}<135 \mathrm{~J}$ and focused through a continuous phase plate (CPP) using an f/6.7 lens producing a 250-micron diameter spot at the center of the plasma. The maximum intensity of the interaction beam was $2 \times 10^{14} \mathrm{~W} \mathrm{~cm}^{-2}$.

The backscattered SBS light was collected and collimated by the focusing lens of the interaction beam. A fraction of the backscattered light was transmitted through the last turning mirror in the laser chain and focused onto a calorimeter. A narrow $1054 \mathrm{~nm}$ band pass filter was used in front of the calorimeter to select out the SBS light.

The third laser beam, $3 \mathrm{~J}$ of $2 \omega\left(\lambda_{2 \omega}=532 \mathrm{~nm}\right)$ laser light in a 2-ns square pulse, was used as a TS probe beam. The interaction beam and the probe beam were timed to turn on 200 ps after the rise in the heater beam. Two TS diagnostics were necessary to measure the absolute frequency of the ion-acoustic waves; the frequency of the TS light in the laboratory frame was shifted from the probe laser frequency by the local frequency of the probed ion-acoustic wave $\left(\omega_{a}=k_{\| \mid} c_{s}\right)$ and by the overall flow $\left(v_{f}\right)$ of the plasma relative to the frame of the laboratory (Doppler shift). The sound speed, flow velocity, driven ion-acoustic wave amplitude and frequency were measured by imaging light scattered from the TS volume onto two separate streak cameras coupled to imaging spectrometers. Both TS diagnostics imaged the same TS volume in the plasma located $400 \mu \mathrm{m}$ towards the interaction beam from the center of the gas jet. The TS volume $\left(70 \times 100 \times 100 \mu \mathrm{m}^{3}\right)$ were defined by the probe beam $(70 \mu \mathrm{m}$ diameter $)$, streak camera slit $(200 \mu \mathrm{m})$, spectrometer slit $(200 \mu \mathrm{m})$, and optical magnification.

The first TS diagnostic provides an independent measurement of the local sound speed through probing ionacoustic fluctuations propagating perpendicular $\left(\mathbf{k}_{\perp}\right)$ to the interaction beam. The spectral and temporal resolution for this system was $0.05 \mathrm{~nm}, 100 \mathrm{ps}$ respectively. The geometry of the second TS diagnostic [Fig. 1a] was chosen to collect light scattered from SBS-driven ion-acoustic waves $\left(k_{\|}\right)$and the counter-propagating thermal ionacoustic fluctuations $\left(-\mathbf{k}_{||}\right)$. The counter-propagating thermal ion-acoustic fluctuations provide a direct measure of the local plasma flow, while the absolute frequency of the SBS-driven ion-acoustic wave $\left(\omega_{\text {driven }}\right)$ is determined using the light scattered from the copropagating ion-acoustic wave. The square root of the

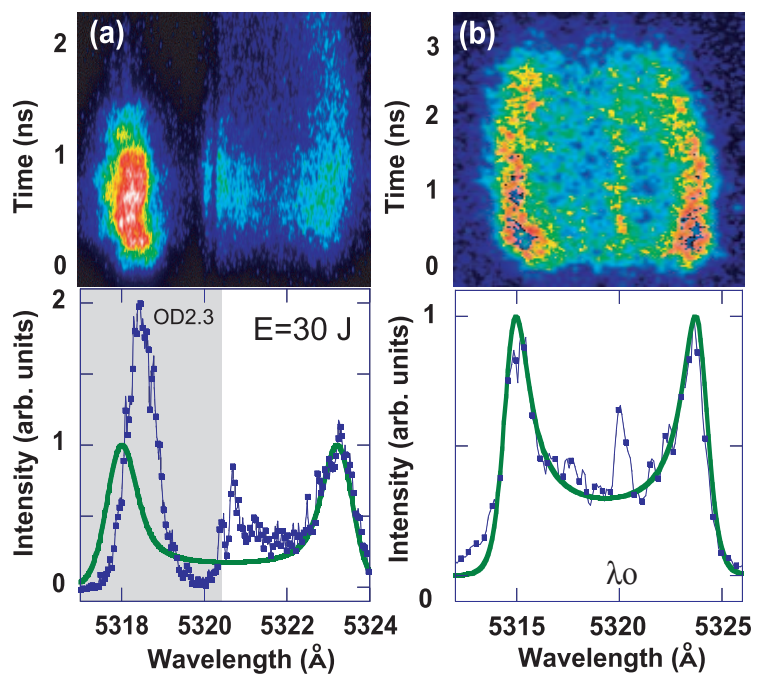

FIG. 2: (color): TS spectra are shown from the (a) SBSdriven diagnostic and (b) thermal diagnostic. The intensity spectra are averaged over 150 ps and are taken 750 ps after the rise of the heater beam once the plasma temperature has settled to a constant value. The standard form factor is plotted on both spectra using an electron temperature of $\mathrm{T}_{e}=350 \mathrm{eV}$ and an ion temperature of $\mathrm{T}_{i}=120 \mathrm{eV}$ (solid line). A velocity flow of $\frac{v_{f}}{v_{t h}}=0.3$ was applied to fit the thermal intensity peak in the SBS-driven spectrum. Comparing the wavelength of the light scattered from the SBS-driven wave to the wavelength of the corresponding form factor peak, gives a wavelength shift of $\Delta \lambda_{T S}=0.6 \AA$. Half of the SBSdriven TS spectrum has been optically filtered by a factor of $\times 1 / 200$.

ratio between the power scattered into the two intensity peaks $\left(P_{\text {driven }} / P_{\text {thermal }}\right)$ is proportional to the ionacoustic wave amplitude $\left(\delta n / n_{e}\right)$. The spectrometer on this diagnostic was run in two modes: in the primary mode the grating was set to second order providing a spectral and temporal resolution of $0.035 \mathrm{~nm}, 100 \mathrm{ps}$ respectively. In the second mode, the grating was set to zero order producing no spectral dispersion but $5.1 \mathrm{ps}$ temporal resolution was achieved. Both TS diagnostics used a narrow 532-nm bandpass filter in front of the spectrometer slit to reject stray light.

Figure 2a shows the direct measurement of a frequency shift $\left(\Delta \lambda_{T S}=0.6 \AA\right)$ of a well-driven ion-acoustic wave. The separation between the intensity peaks in the form factor is determined by the sound speed which was measured independently [Fig. 2b]. Comparing the frequency of the driven intensity peak $\left(\omega_{\text {driven }}\right)$ with the corresponding resonant frequency given by the form factor, provides a direct measure of the local frequency shift, $\Delta \omega / \omega_{a}=-\Delta \lambda_{T S} / \lambda_{2 \omega}$, given by,

$$
\Delta \omega=\left(\omega_{\text {driven }}-\omega_{2 \omega}\right)-\left(\omega_{a}+\mathbf{k}_{||} \cdot \mathbf{v}_{\mathbf{f}}\right) .
$$

Figure $2 \mathrm{~b}$ shows data collected from the thermal TS diagnostic $\left(\mathbf{k}_{\perp}\right)$ that provides a direct measure of the 


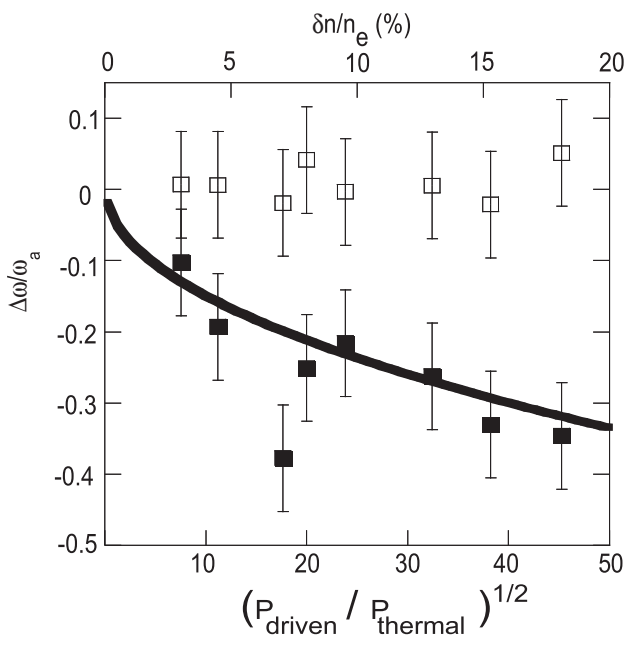

FIG. 3: The frequency of the SBS driven ion-acoustic wave (solid squares) decreases as the amplitude of the wave increases. The frequency of the thermal fluctuations (open squares) remains relatively constant indicating that the interaction beam has little effect on the plasma. The solid line corresponds to Eq. 1 where the ion-acoustic wave amplitude (upper axis) is assumed to be proportional to the square root of the ratio of the amplitude of the two TS peaks.

ion-acoustic sound speed. By fitting the standard theoretical form factor, a measure of the electron and ion temperatures can be inferred; the ion temperature is determined by fitting the spectral width of the two ionacoustic peaks, $\mathrm{T}_{i}=120 \pm 50 \mathrm{eV}$ at $750 \mathrm{ps}$. The electron temperature is given by the separation between the two peaks in the thermal TS diagnostic, $\mathrm{T}_{e}=350 \pm 50 \mathrm{eV}$ at $750 \mathrm{ps}$ [15], which agrees well with hydrodynamic simulations that were done with the code HYDRA [16] using the measured density profile. The errors in the temperature measurements were determined by changing the respective parameters to fit the form factor to the extremes of the noise in the data. The electron temperature was measured to be independent of the interaction beam intensity within the measurement accuracy $( \pm 15 \%)$.

Figure 3 shows the ion-acoustic frequency decreasing $\left(\Delta \omega / \omega_{a}=-0.1\right.$ to -0.35$)$ as the amplitude of the SBSdriven ion-acoustic wave increases by an order of magnitude. Given our plasma conditions, with $\mathrm{ZT}_{e} / T_{i}<10$, ions can be easily trapped in the potential energy well created by the large driven ion-acoustic waves. These trapped ions create a frequency shift in the local SBS driven ion-acoustic wave; this frequency shift detunes the SBS instability, saturating the ion-acoustic wave growth. The resulting frequency shift can be calculated given the amplitude of the ion-acoustic wave [8-10]:

$$
\frac{\Delta \omega}{\omega_{a}} \approx-\frac{1}{3} x^{4} \sqrt{\frac{Z T_{e}}{T_{i}}} e^{-x^{2} / 2} \sqrt{\frac{\delta n}{n_{e}}} \equiv-\eta\left(\frac{\delta n}{n_{e}}\right)^{1 / 2}
$$

where $x=c_{s} / v_{t h} \approx \sqrt{Z T_{e} / T_{i}+3}$ and $\eta$ is the detuning
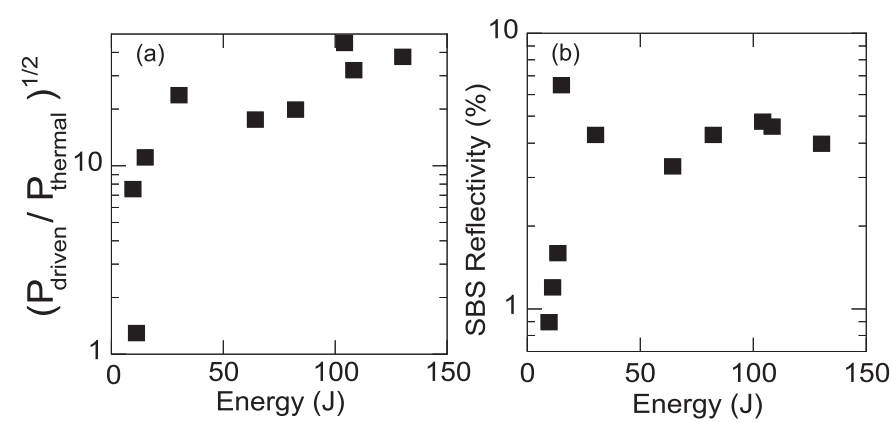

FIG. 4: (a) The amplitude of the SBS-driven ion-acoustic wave and (b) the SBS reflectivity is shown to saturate at an interaction beam energy of $30 \mathrm{~J}$ corresponding to an ionacoustic wave amplitude of $\Delta n / n_{e}=0.07$ as shown in Fig. 3 .

parameter equal to -0.75 calculated for our conditions.

The ion-acoustic wave amplitude $\left(\delta n / n_{e}\right)$ can be calculated from the intensity peaks in the TS spectra by comparing the scattered power from the driven acoustic wave $\left(\mathrm{P}_{\text {driven }}\right)$ with the power scattered from the thermal fluctuations $\left(\mathrm{P}_{\text {thermal }}\right)[17,18]$ :

$$
\frac{P_{\text {driven }}}{P_{\text {thermal }}}=n_{e} \lambda_{2 \omega}^{2} \Delta \Omega_{\text {exp }} L_{c}\left(\frac{\delta n}{n_{e}}\right)^{2}
$$

where $L_{c}$ is the correlation length along the direction of the TS probe beam, and $\Delta \Omega_{\text {exp }}$ is the solid angle of the collection optics $(f / 5$ lens $) . L_{c}$ can be estimated by the transverse size of speckles generated by the interaction beam $f_{1 \omega} \lambda_{1 \omega} \sim 5 \mu \mathrm{m}$. Using the measured parameters inside the TS volume, Eq. 2 leads to ion-acoustic wave amplitudes $\left(\delta n / n_{e}\right)$ that are a factor of 3 lower than those plotted in Fig. 3 which were obtained by fitting the data to Eq. 1 and assuming $\left(\frac{\delta n}{n_{e}}\right)^{2} \propto P_{\text {driven }} / P_{\text {thermal }}$. The fact that Eq. 2 underestimates the amplitude of the wave is likely due to the fact that our diagnostic is averaging over several speckles in the TS volume, the uncertainties in estimating $\mathrm{L}_{c}$, and the possibility of enhanced thermal fluctuations as previously reported [7, 19, 20].

We have further correlated the measured increasing frequency shift with the saturation of the SBS instability. Figure $4 \mathrm{a}$ shows that the local amplitude of the driven ion-acoustic wave is saturated for energies above $\mathrm{E}=30 \mathrm{~J}$ and wave amplitudes above $\delta n / n_{e}=0.07$. Figure $4 \mathrm{~b}$ shows, as expected, that the SBS reflectivity saturates at the same laser intensity as the ion-acoustic waves. The observation of a correlated increase in the frequency shift [Fig. 3] is strong evidence that trapping induced frequency shifts are responsible for the saturation of SBS. Furthermore, for our conditions the linear gain is $\mathrm{G}_{s b s}>20$ predicting a reflectivity near $100 \%$ for a scale-length of $1-\mathrm{mm}$. In order to match the measured reflectivity of $5 \%$, the nonlinear frequency shift is effectively reducing the scale-length used in calculating the linear gain by at least factor of three [21]. 


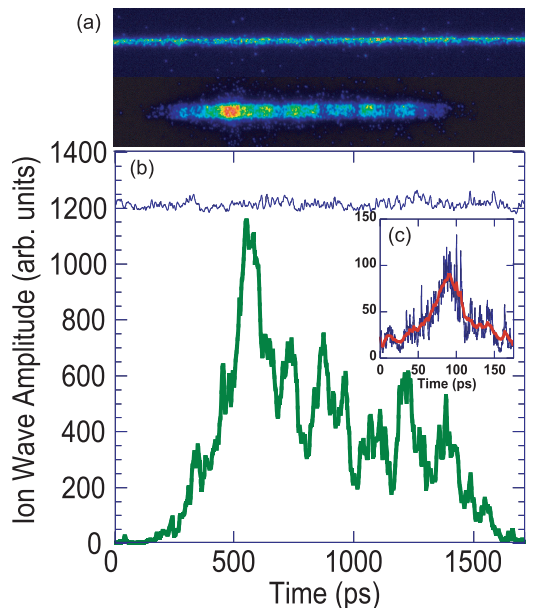

FIG. 5: (color): (a) Raw 2-ns streak data with 35 ps temporal resolution are shown for both the incident TS probe beam (top) and the scattered TS signal from the SBS-driven ion-acoustic wave (bottom) with an interaction beam energy of $\mathrm{E}=50 \mathrm{~J}$. (b) Fast ion-acoustic wave amplitude modulations are shown in the intensity profile from the image above (thick green line). The TS signal is evident only while the ionacoustic wave is being driven by the interaction beam. The amplitude of the interaction beam was flat to within $10 \%$ over the 1.2-ns. There are only small modulations in the incident probe beam (thin blue line). (c) A $28 \pm 5$ ps amplitude modulation was time resolved near the middle of the interaction beam pulse using a 170 ps temporal window with 5.1 ps resolution.

Due to the measured detuning in the SBS three-wave process, fast $30 \mathrm{ps}$ amplitude modulations in the driven ion-acoustic wave have been measured [Fig. 5]. These fast modulations have been predicted by various SBS simulations $[10,21,22]$. The measured modulations are consistent with the measured frequency shift reported in Fig. 3 $\left(\Delta \omega / \omega_{a}=-0.2\right)$, which gives a time scale for amplitude modulations of $\Delta t=2 \pi / \Delta \omega=15 \mathrm{ps}$.

In order to measure the temporal (ps) modulations in the amplitude of the ion-acoustic wave, the streak camera and spectrometer slits were closed to 50 microns and 100 microns respectively to reduce spatial averaging by limiting the TS volume to $70 \times 25 \times 50 \mu \mathrm{m}^{3}$. Furthermore, the spectrometer grating was set to zero order to limit temporal dispersion. Using $2 \omega 500$ fs laser pulses, the temporal response of the system was measured to be better than 30 pxls on each time scale of the streak camera, corresponding to 5.1 ps on the fastest sweep speed.

These measurements of large amplitude ion-acoustic wave modulations indicate that the SBS instability can be driven out of the linear regime even when the average (time and space) reflectivity suggests that a linear model is adequate.

In summary, we have presented the first direct observation of the saturation of SBS by an ion-trapping induced frequency shift. The measured frequency shift in the driven ion-acoustic wave increases by a factor of 3 when the amplitude of the wave increases by over an order of magnitude, which is in good agreement with theoretical calculations of the nonlinear frequency shift induced by ion trapping. Furthermore, the measured frequency shift was correlated with fast amplitude modulations in the SBS-driven ion-acoustic wave, as observed in numerous numerical simulations. These findings confirm the importance of kinetic effects in limiting the growth of ion-acoustic waves in low-Z plasmas and will help understand and control the SBS reflectivity on future ICF indirect-drive experiments.

We would like acknowledge the efforts of the Janus laser crew in performing a successful upgrade. We thank S. Dixit, M. Rushford, C. Hoaglan, and Mike Aasen for the design and quick fabrication of the CPP. Furthermore, we thank R. Griffith for the streak camera support and R. Rieger of Roper Scientific for the instrument loan. We also acknowledge motivating discussions on the physics of particle trapping with $\mathrm{B}$. Cohen and E. Williams. This work was performed under the auspices of the U.S. Department of Energy by the Lawrence Livermore National Laboratory under Contract No. W7405-ENG-48.

* Department of Electrical Engineering, University of Alberta, Edmonton, Alberta T6G 2V4, Canada

$\dagger$ Also at University of British Columbia, Vancouver, B.C. Canada

₹ Also at Department of Applied Science, University of California at Davis, 95616.

[1] J. D. Lindl et al., Phys. Plasmas 11, 339 (2004).

[2] H. Baldis et al., Phys. Rev. Lett. 80, 1900 (1998).

[3] W. Seka et al., Phys. Rev. Lett. 89, 175002 (2002).

[4] H. A. Rose and D. F. DuBois, Phys. Rev. Lett. 72, 2883 (1994).

[5] R. L. Berger et al., Phys. Plasmas 5, 4337 (1998).

[6] A. Ng et al., Phys. Rev. Lett. 42, 307 (1979).

[7] S. H. Glenzer et al., Phys. Rev. Lett. 86, 2565 (2001).

[8] D. H. Froula et al., Phys. Rev. Lett. 88, 105003 (2002).

[9] G. J. Morales and T. M. O'Neil, Phys. Rev. Lett. 28, 417 (1972).

[10] B. I. Cohen et al., Phys. Plasmas 4, 956 (1997).

[11] E. A. Williams et al., Phys. Plasmas 11, 231 (2004).

[12] J. Handke et al., Phys. Rev. Lett. 51, 1660 (1983).

[13] S. Semushin and V. Malka, Rev. Sci. Instrum. 72 (2001).

[14] Y. M. Li and R. Fedosejevs, Meas. Sci. Technol. 5, 1197 (1994).

[15] S. D. Baton et al., Physical Review E 57 (1998).

[16] M. Marinak et al., Phys. Plasmas 5, 1125 (1998).

[17] P. E. Young, Phys. Rev. Lett. 73 (1994).

[18] J. Meyer and H. Houtman, Phys. Rev. Lett. 53 (1984).

[19] C. E. Clayton et al., Phys. Rev. Lett. 51, 1656 (1983).

[20] D. H. Froula et al., Phys. Rev. Lett. 90, 155003 (2003).

[21] L. Divol et al., Phys. Plasmas 10, 1822 (2003).

[22] A. A. Andreev and V. T. Tikhonchuk, JETP 68, 1135 (1989). 